\title{
Pharmacist Intervention with Computer-Based Alert System and Assessment of Prescription Errors for Outpatient in a Chinese Tertiary Hospital
}

\author{
Qing Shen, ${ }^{a, b}$ Yi-Meng Zhou, ${ }^{a, b}$ Jin Lv, ${ }^{a, b}$ Hua Yang, ${ }^{a, b}$ Yuan-yuan He, ${ }^{a, b}$ Yuan Bian, ${ }^{a, b}$ and Yong Han ${ }^{*, a, b}$ \\ ${ }^{a}$ Department of Pharmacy, Sichuan Academy of Medical Sciences \& Sichuan Provincial People's \\ Hospital/Affiliated Hospital of University of Electronic Science and Technology of China, Chengdu, \\ Sichuan 610072, Sichuan, China \\ ${ }^{b}$ Personalized Drug Therapy Key Laboratory of Sichuan Province, School of Medicine, University of Electronic \\ Science and Technology of China, Chengdu, Sichaun 610072, China
}

Email: 532317646@qq.com (Y. H.)

\begin{abstract}
Qualified prescribing is fundamental for appropriate use of medicine. Pharmacist interventions potentially remind physicians to cope with this issue. This study aimed to report interventions implemented by pharmacists with real-time computer-based alert system and evaluate the prescription errors for outpatients in a Chinese tertiary hospital. This retrospective, non-comparative study was implemented with an analysis of data collected from October to December in 2018 involved all clinical specialty departments in the hospital. Alerts were generated and displayed on pharmacist screen while prescribing inappropriately, then pharmacists chose certain operation and offered recommendations according to the alerts. Outcomes such as category and acceptance rate of pharmacist recommendations, prescription error types, prevalence rate, severity level and monthly tendency were documented, categorized and analyzed. In our study, $2.3 \%$ of prescriptions generated alerts, and incidence of pharmacist interventions was $0.19 \% .431$ prescription errors were prevented, with the overall acceptance rate of interventions accounted for $29.7 \%$. Dosage or frequency problems $(n=170,39.5 \%)$, contraindications $(n=79,18.3 \%)$ and improper duration of pharmacotherapy $(n=53,12.3 \%)$ were the top three types of prescription errors. Monthly rate of errors decreased from 0.98 to 0.43 per 1000 prescriptions in the monitoring period. Pharmacist interventions with computer-based alert system were available for filtering part of invalid alerts and preventing prescription errors in our study.
\end{abstract}

Keywords pharmacist intervention, computer-based alert system, outpatient, prescription review

\section{Introduction}

A licensed pharmacist initiates any proposal of action, which may result in physician's intention to change the management or therapy of patient is defined as Pharmacist Intervention $(\mathrm{PI}) .{ }^{[1]}$ In initial period, in order to maximize the benefits while minimizing clinical risks, pharmacists provided information for physicians by means of rational communication, which was suitable to design more achieving therapeutic regimen. ${ }^{[2]}$ Nowadays, pharmacists play an important role in medication reconciliation associated with reduced discrepancies, improved accuracy and decreased mortality. ${ }^{[3]} \mathrm{A}$ systematic review ${ }^{[4]}$ showed that prescription errors associated with computerized physician order entry (CPOE) system ranged from $6.1 \%-77.7 \%$, becoming the most common medication errors. ${ }^{[5]}$ Alsaidan ${ }^{[6]}$ reviewed 9 studies focused on $\mathrm{PI}$ in the Gulf cooperation Council countries. One of these indicated that clinical pharmacists prevented $52 \%$ prescribing errors. In addition, the intervention rate on drug level and prescriber level were approximately $43 \%$ and $40 \%$, respectively, ${ }^{[7]}$ most of which were preventable. The percentage of intervention acceptance ranged from 58\% up to approximately $98 \%$ in these researches.

Computer-based alert system (CAS) integrated into CPOE, which was used in controlling potential duplicated medication, drugs interaction and the like, can reduce prescription errors in some degree. ${ }^{[8-10]}$ Zenziper group ${ }^{[11]}$ found improper prescriptions were reduced by $50 \%$ through customized alerting tool. However, the system often operated with low sensitivity and specificity, generating clinical invalid alerts which brought about "alert fatigue", and the compliance of prescriber was decreased inevitably. ${ }^{[12]}$ In these circumstances, we conducted a retrospective study with respect to $\mathrm{PI}$ based on CAS.

In China, different from other countries in the world, PI has been compulsive since 2018 after laws and regulations were issued by National Health Commission. Pharmacists reviewed prescriptions during dispensing of medicine for outpatient before. The procedure was troublesome and complex under such circumstances once prescription error was found. Although computer-assisted alert tool for medication is globally used, limited information concerns PI based on CAS about outpatient prescription is available in China.

With the aim of describing pharmacist intervention with real-time CAS in interactive mode which turned passive surveillance to active monitoring, ${ }^{[13]}$ we introduced the progress and analyzed prescription errors in this study.

\section{Materials and Methods}

\section{Study setting and computer-based alert system}

The intervention we described retrospectively was implemented in a Chinese tertiary hospital. The staffs associated with $\mathrm{PI}$ comprised four clinical pharmacists, registered outpatient physicians, and a software maintainer. $A$ tool in CAS named PASSpharmReview (Wolters Kluwer/ Medicom software. Ltd., Chengdu, Sichuan, China) for intervention was incorporated into Hospital Information System (HIS), which was similar to CPOE and available for prescribing. 
The tool was programmed with C\# language, in which 190672 drugs information with more than 10 million algorithms were found included to produce alerts and intercept potential prescription errors. What's more, alarm information was displayed on pop-up window and viewed by pharmacist through Google chrome. The alert was triggered at which severity level could be defined by clients, although what level the error vested in was defined by system. It was ranked of three kinds of color including orange, red and black with the severity level escalated.

\section{Study design}

A non-comparative, descriptive, observational study was performed. Data of prescription errors for analysis was retrospectively collected from October to December in 2018. Pharmacist intervention service involved in this article was provided $8 \mathrm{~h}$ a day, $5 \mathrm{~d}$ a week. This study aimed to evaluate the PI and analyze the prescription errors in a tertiary hospital of China. About 90 clinical specialties of outpatient were enrolled in this study. The prescription information was excluded out of the statistics when pharmacists weren't on line for PI. Nothing else was implemented for exclusion. PI tool was encrypted to prevent patients' information from disclosing.

\section{Pharmacist intervention}

Every outpatient physician's order was transmitted to the real-time CAS to guarantee that all PIs were proactive. The flowchart of PI based on CAS and physician's behavior was described in Figure 1. The same protocols were adopted for intervention, including instructions from manufacture, domestic and exotic evidenced clinical guidelines, national and international pharmacotherapy criteria. Alerts were assigned to different pharmacist equably without repetition even though they were on line simultaneously. The monitored items included indications, contraindications, special population, drug selection, drug-therapy duplication, dosage, drug-treatment duration, and drug-drug interaction. When an alert took place, pharmacists could read patient baseline characteristics and medication information such as generic names, trade names, usage, dosage, drug quantity, severity levels and recommendations displayed on the window.

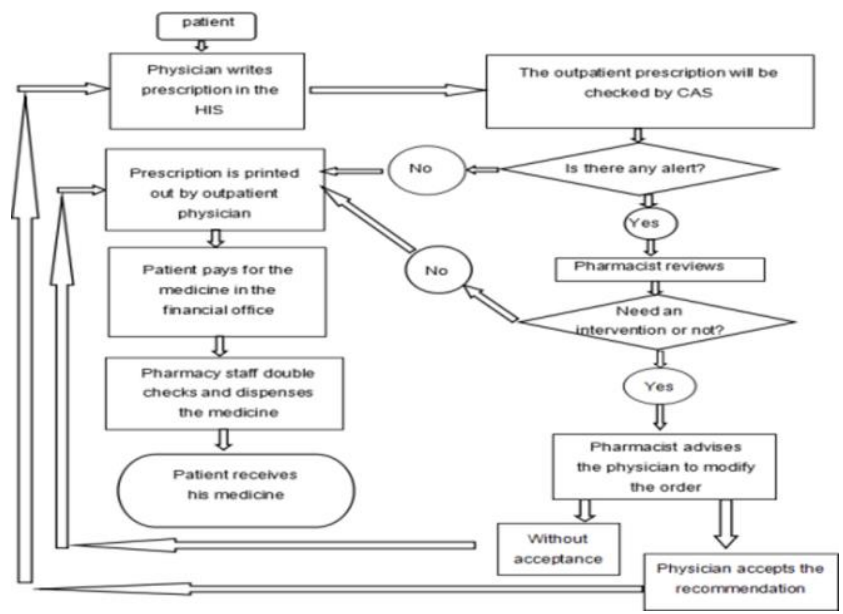

Figure 1 Flow diagram of outpatient prescription reviewing from order producing to drug administration in a Chinese tertiary hospital. HIS, hospital information system; CAS, computer alert system.

There were customized $1 \mathrm{~min}$ and system defined 3 choices for pharmacists to deal with the alert: (1) override the alert (2) determined by physician (3) need a modification from physician.
The definition of options available on the screen was detailedly shown in Table S1. Pharmacists could communicate with physician through a dialog box in the tool to give recommendations. Interventions implemented by pharmacists and physicians' response were recorded by CAS for summarizing. Type, PI rate and the acceptance rate were evaluated.

\section{Assessment of prescription errors}

In this study, the errors only referred to prescribing inappropriately for outpatient. If the prescriber accepted the recommendation from pharmacist and changed the order, the alert was scored as a prescription error. ${ }^{[14]}$ Otherwise, the prescription was regarded as rational when physician refused to modify the order. Prescription error data was collected and documented, then evaluated in terms of prevalence rate, category of medication, error type, related clinical specialties and severity level.

\section{Data collection and analysis}

After the pharmacist processed alerts, the result was saved automatically. The data was collected in Microsoft Excel 2010 (Microsoft Company, WA, USA) by writer and subsequently summarized for analysis. Ratio and corresponding $95 \% \mathrm{Cl}$ (confidence interval) for acceptance rate of $\mathrm{PI}$ were calculated. Data statistic was implemented with SPSS 18.0 (IBM, Chicago, IL, USA).

This work was approved by the research ethics committee of Sichuan Provincial People's Hospital (No. 2019-198).

\section{Results}

\section{Pharmacist intervention}

During the study period of three months, 755088 prescriptions were collected from HIS. 17115 ones with the percentage of $2.3 \%$ generated alerts, and $0.19 \%$ of prescriptions required pharmacist intervention in the monitoring period (1450 of 755088).

PI referred to giving medication advice and needing a confirmation from prescriber in this article. In total of 431 recommendations with the percentage of $29.7 \%$ were accepted by physicians. Changing the dosage or frequency of drug occurred most frequently (Table S2). In the overall recommendations, adding indication was well accepted by physicians which accounted for $94.4 \%$, although the incidence rate was as low as $3.7 \%$. Changing in drug total quantity was neglected by most physicians with the reason to be explored. The trend of $\mathrm{PI}$ rate (pharmacists intervention number per month/total prescription number per month ${ }^{*} 1000 \%$ ) declined from $2.79 \%$ (627 of 224802) in October to $1.92 \%$ (453 of 235380 ) in November and 1.63\% (370 of 226996) in December during the study period.

\section{Assessment of prescription errors}

A prescription error would emerge with the confirmation of both pharmacist and physician. Among the prevented errors, there were 223 male and 208 female patients with the mean age of $57.2 \pm 21.1$ years, respectively. $96(22.3 \%)$ ones who encountered the vast majority of medication recommendations were treated with neoplasm surgery. Besides, a total of 56 (13.0\%) patients suffered from renal insufficiency. Among all prescription errors, a large proportion of them were concerned with traditional Chinese patent medicine or extraction (45.9\%). Contraindication contributed a proportion of $18.3 \%$ (79 of 431 ), which was less than half the rate of irrational drug dosage or frequency (Figure 2). We noted oral medicine as drugs most often associated with prescription errors (363 of $431,84.2 \%$ ), followed by injections ( 55 of $431,12.8 \%$ ), and the medicine for 


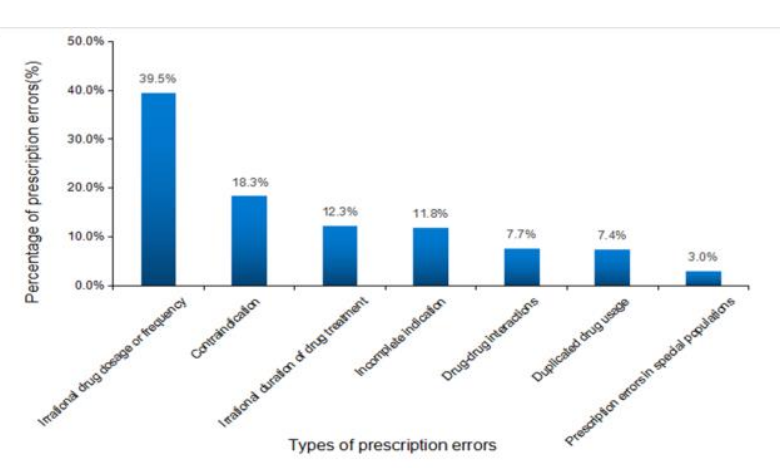

Figure 2 Percentage of prescription errors in different types for outpatient. "Special populations" referred to pregnancy, lactation and children.

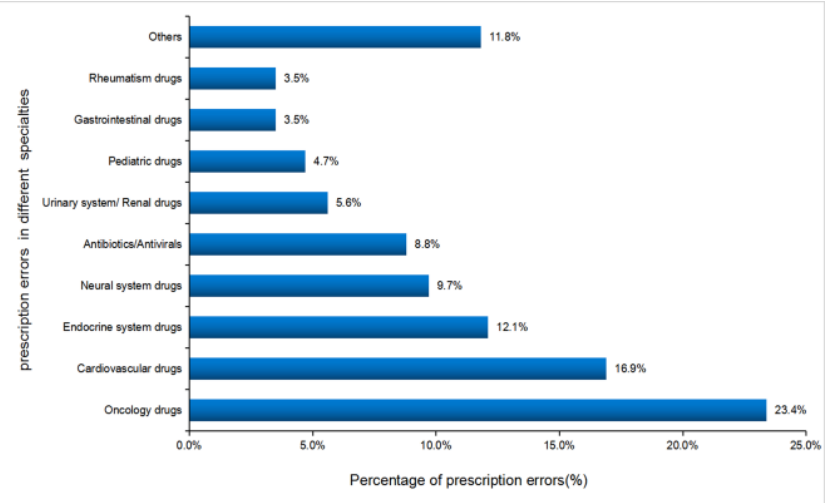

Figure 3 Percentage of prescription errors encountered in different clinical specialties "Others" referred to hepatinica, anticoagulant, anti-asthmatics, dermatological drugs, ophthalmic drugs. "Oncology drugs" referred to anti-neoplastic and adjuvant.

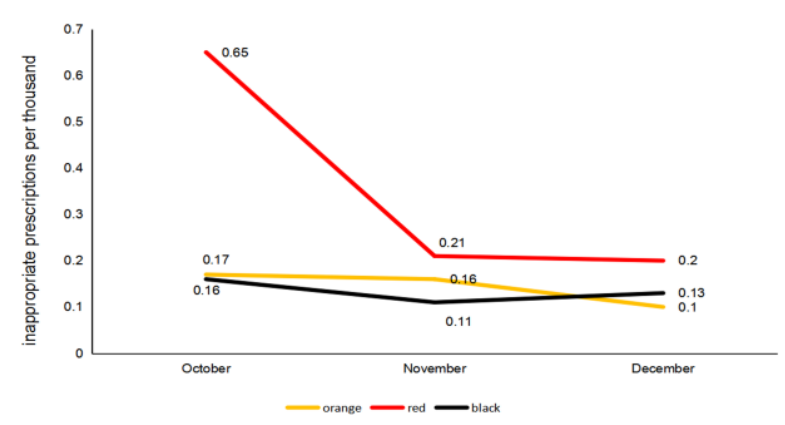

Figure 4 Monthly rate of prescription errors in different severity level per 1000 prescriptions. Every error was labeled with a level automatically when alerted. Monthly rate of prescription error $=$ prescription error number in each level/ total prescription number each month * $1000 \%$.

external use (13 of $431,3.0 \%$ ). Prescription errors concerned drug categories were displayed (Figure 3 ). As a result, the trend of prescription errors rate (prescription error number per month/ total prescription number per month * $1000 \%$ ) declined from $0.98 \%$ in October to $0.48 \%$ o in November and $0.44 \%$ in December in the study period (Figure 4).

\section{Discussion}

Pharmacists from China face significant challenge and need a transition from traditional drug dispensing to clinical work participation along with the revolution of Chinese health care nowadays. With the promulgation of regulations for prescription reviewing, much attention was paid to $\mathrm{PI}$ by the relevant department of our state. This research gained an insight into the significance of outpatient pharmacy service in clinical focused mode.

In our records, prescriptions which generated alerts accounted for $2.3 \%$. By contrast, the proportion of orders triggered alerts varied from $0.4 \%$ to $30.1 \%$ in some researches. ${ }^{[9-11,15]}$ These outcomes were varied, which was probably attributed to the category of supervision regime, prescribing habit of physician and the set of alert threshold. The prevalence of $\mathrm{PI}$ was $0.19 \%$, different from traditional CDSS (clinical decision support system), clinical invalid alerts were filtered by pharmacist in this study. Only one-minute interval was customized for providing recommendation followed by physician's choice, so that specific clinical skills which required further improvement for pharmacotherapy were essential. Pharmacists and physicians overrode alerts, which were estimated benefit of treatment outweighed risks, or classified as low severity level with few evidence. Pharmacists had the permission for "hard stop" for fatal alerts. ${ }^{[16]}$

The top three frequent prescription errors were dosage or frequency problems, contraindications, duration of pharmacotherapy in our study. Variation from region, age, and clinical specialties, the outcomes presented differently. Contraindications mostly referred to patients, who suffered from renal insufficiency or epilepsy. Thus, adjustment of dose or frequency was required for drugs eliminated by renal system. Replacing drugs which were considered high risky to cause rhabdomyolysis was necessary for epileptics whose symptom were not well controlled. Medication for patients with more than two indications was more complex, especially for drug metabolism disordered ones. Physicians were skilled in prescribing drugs used in their own specialty, but they might be unfamiliar with drugs used in other subjects. ${ }^{[14]}$ Patients with chronic disease needed long-term drug therapy, therefore, the period of treatment was considerable. Dosage adjustment was required under certain conditions after blood concentration monitoring, as well as liver function test.

Anti-neoplastic drugs and the adjuvant, cardiovascular drugs, endocrine system drugs, which were prescribed and then modified by physicians most frequently, presented higher risk of errors. As a kind of oncology medicine, traditional Chinese medicine (TCM) could reduce toxicity and increase efficacy when combined with chemotherapy, playing a role in maintenance therapy for improving life quality of malignant tumor patients. ${ }^{[17-19]}$ However, as mixed compounds, there was drug-drug interaction between particular ingredients of different Chinese patent medicine. This situation reported by Chen ${ }^{[20]}$ also existed extensively among TCM and western drugs despite widespread use in Taiwan, China. Recommendations of replacing the secondary drug or minimizing the dosage was required to reduce potential risks.

The overall acceptance rate of PI was 29.7\%. Recommendations for incomplete indication were most acceptable, most of which were due to inadvertence of prescribers. Advice of irrational drug usage in special population was second popular when pharmacists mentioned what certain medicine could replace it. Physicians accepted recommendations of drugs substitute more often than removing them occasionally when came across drug-drug interaction with the acceptance rate of $32.4 \%$, higher than $8.5 \%$ from Yeh ML's study. ${ }^{[10]}$ Physicians' compliance was found related to the method of alarming. The pop-up alerts were reported more accepting while physician was not in the workflow of prescribing. ${ }^{[21]}$ Due to the process was delayed by intervention, 
which might cause physician's anxiousness and negative attitude towards this system. Recommendations were treated as increased burden and skipped without any response somewhile. A systematic review ${ }^{[22]}$ summarized that alert burden, low sensitivity, low clinical relevance, unnecessary disruptions led to ignorance of alerts. Nevertheless, pharmacist involvement in medication reconciliation could stop a part of clinical useless alerts and provide medication recommendation for physician through dialogue box. This practice increased the acceptance rate and improved practitioner performance even though the delay still existed. Horsky ${ }^{[16]}$ reported there were trade-offs for clinicians to evaluate risks for individual patient. Recommendations which were considered out of clinical value were ignored, to which the current study of pharmacist reviewing based on CAS was similar. High severity level alerts scarcely generated, physicians probably associated it with high risk to avoid while prescribing. Low severity level alerts with advice were ignored when considered less risky and the therapeutic regimen was treated more beneficial for patient.

CAS was reported to have the function of reducing medication errors and improving prescribing behavior in different population and clinical territory. ${ }^{[12,13,16,23,24]}$ Our study was compatible with this. Large quantity of appointments with limited time formed one feature of outpatient service in Chinese hospital, therefore, a well customer defined CAS can make a progress in improving efficiency for both reminders and physicians. Further refinement was required to reduce alerts fatigue $^{[16]}$ and improve clinical relevance ${ }^{[25]}$

On account of investigating computer-based pharmacist intervention for outpatient in the mainland of China was in initial stage, there were some limitations in this study. First, the information of medical records for outpatients wasn't exhaustive enough for pharmacist reviewing what could lead to uncalled-for Pls. Pharmacists could only get feedback from physicians when $\mathrm{Pl}$ occurred which wasn't comprehensive enough to make more appropriate recommendations. Second, on account of this non-comparative study we conducted, only descriptive statistics without control group was addressed. Inferential statistical method may be more evidential in further studies. Third, digital medication reviewing couldn't substitute for manual reviewing, ${ }^{[26]}$ and a combination of both can compensate for the vulnerability of system. Not every prescription error could be alerted based on criterion set by system. Embedding standards in reviewing software artificially should lower the false negative rate of alerts which called for further research.

\section{Conclusions}

PI based on CAS and retrospective prescription error analysis which partly presented a reduction in incidence rate was implemented in our study. 431 potential errors were probably prevented before harms reached the patients. PI could filter a portion of invalid alerts, and the overall acceptance rate of $\mathrm{PI}$ accounted for $29.7 \%$. The analysis demonstrated that dosage or frequency problems, contraindications, and improper duration of pharmacotherapy were the top three frequent types of errors. Oncology drugs followed by cardiovascular drugs encountered the largest number of recommendations in our study. These outcomes revealed a perspective to understand prescription error of outpatient in China and provided reference material for us to improve clinical pharmacy service quality in the coming days.

\section{Acknowledgments}

All the authors gratefully appreciated Wolters Kluwer/ Medicom company for providing intervention software and data maintenance. Other thanks were due to all colleagues who participated in our study.

\section{Supporting Information}

Supporting information for this article is available on the WWW under www.genchemistry.org/EN/10.21127/yaoyigc20210009.

\section{Conflict of Interest}

The authors declare no conflict of interest.

Copyright (C) 2021 Qing Shen, Yi-Meng Zhou, Jin Lv, Hua Yang, Yuan-Yuan He, Yuan Bian, and Yong Han. This article is an open access article distributed under the terms and conditions of the Creative Commons Attribution (CC BY) license (http://creativecommons.org/ licenses/by/4.0/). The use, distribution or reproduction in other forums is permitted, provided the original author(s) or licensor are credited and that the original publication in this journal is cited, in accordance with accepted academic practice. No use, distribution or reproduction is permitted which does not comply with these terms.

\section{References}

[1] Vo, T. H.; Bardet, J.-D.; Charpiat, B.; Leyrissoux, C.; Gravoulet, J.; Allenet, B.; Conort, O.; Bedouch, P. Validation of a tool for reporting pharmacists' interventions in everyday community pharmacy. J. Clin. Pharm. Ther. 2018, 43, 240-248.

[2] Hawkins, D. W. Clinical Pharmacy Functions in Ambulatory Patient Care. J. Clin. Pharmacol. 1981, 21, 245-250.

[3] Splawski, J.; Minger, H. Value of the Pharmacist in the Medication Reconciliation Process. P\&T 2016, 41, 176-178.

[4] Korb-Savoldelli, V.; Boussadi, A.; Durieux, P.; Sabatier, B. Prevalence of computerized physician order entry systems-related medication prescription errors: A systematic review. Int. J. Med. Inform. 2018, 111, 112-122.

[5] Bosma, B. E.; Bemt, P. M. L. A. v. d.; Melief, P.; Bommel, J. V.; Hunfeld, N. Pharmacist interventions during patient rounds in two intensive care units: Clinical and financial impact. Neth. J. Med. 2018, 76, 115-124.

[6] Alsaidan, J.; Portlock, J.; Aljadhey, H. S.; Shebl, N. A.; Franklin, B. D. Systematic review of the safety of medication use in inpatient, outpatient and primary care settings in the Gulf Cooperation Council countries. Saudi. Pharm. J. 2018, 26, 977-1011.

[7] Kheir, N.; Awaisu, A.; Sharfi, A.; Kida, M. Adam A. Drug-related problems identified by pharmacists conducting medication use reviews at a primary health center in Qatar. Int. J. Med. Inform. 2014, 36, 702-706.

[8] Yang, C. Y.; Lo, Y. S.; Chen, R. J.; Liu, C. T. A Clinical Decision Support Engine Based on a National Medication Repository for the Detection of Potential Duplicate Medications: Design and Evaluation. JMIR Med. Inform. 2018, 6, e6.

[9] Segal, G.; Segev, A.; Brom, A.; Lifshitz, Y.; Wasserstrum, Y.; Zimlichman, E. Reducing drug prescription errors and adverse drug events by application of a probabilistic, machine-learning based clinical decision support system in an inpatient setting. $J$. Am. Med. Inform. Assoc. 2019, 26, 1560-1565.

[10] Yeh, M. L.; Chang, Y. J.; Wang, P. Y.; Li, Y. C.; Hsu, C. Y. Physicians' responses to computerized drug-drug interaction alerts for outpatients. Comput. Meth. Prog. Bio. 2013, 111, 17-25.

[11] Zenziper, Y.; Kurnik, D.; Markovits, N.; Ziv, A.; Shamiss, A.; Halkin, H.; Loebstein, R. Implementation of a clinical decision support system for computerized drug prescription entries in a large tertiary care hospital. I. M. A. J. 2014, 16, 289-294.

[12] McCoy, A. B.; Thomas, E. J.; Krousel-Wood, M.; Sittig, D. F. Clinical decision support alert appropriateness: a review and proposal for improvement. Ochsner. J. 2014, 14, 195-202. 
[13] Chen, C.; Jia, W.; Guo, D.; Zhu, M.; Xu, Y.; Wang, X.; Wang, D.; Wang, W.; Tang, Z. Development of a Computer-Assisted Adverse Drug Events Alarm and Assessment System for Hospital Inpatients in China. Ther. Innov. Regul. Sci. 2020, 54, 32-41.

[14] Jiang, S. P.; Chen, J.; Zhang, X. G.; Lu, X. Y.; Zhao, Q. W. Implementation of pharmacists' interventions and assessment of medication errors in an intensive care unit of a Chinese tertiary hospital. Ther. Clin. Risk. Manag. 2014, 10, 861-866.

[15] Sethuraman, U.; Kannikeswaran, N.; Murray, K. P.; Zidan, M. A.; Chamberlain, J. M. Prescription Errors Before and After Introduction of Electronic Medication Alert System in a Pediatric Emergency Department. Acad. Emerg. Med. 2015, 22, 714-719.

[16] Horsky, J.; Aarts, J.; Verheul, L.; Seger, D. L.; van der Sijs, H.; Bates, D. W. Clinical reasoning in the context of active decision support during medication prescribing. Int. J. Med. Inform. 2017, 97, 1-11.

[17] Liu, Z. L.; Zhu, W. R.; Zhou, W. C.; Ying, H. F.; Zheng, L.; Guo, Y. B.; Chen, J. X.; Shen, X. H. Traditional Chinese medicinal herbs combined with epidermal growth factor receptor tyrosine kinase inhibitor for advanced non-small cell lung cancer: a systematic review and meta-analysis. J. Integr. Med. 2014, 12, 346-358.

[18] Han, Y.; Wang, H.; Xu, W.; Cao, B.; Han, L.; Jia, L.; Xu, Y.; Zhang, Q.; Wang, X.; Zhang, G.; Yu, M.; Yang, G. Chinese herbal medicine as maintenance therapy for improving the quality of life for advanced non-small cell lung cancer patients. Complement. Ther. Med. 2016, 24, 81-89.

[19] Dong, J.; Su, S. Y.; Wang, M. Y.; Zhan, Z. Shenqi fuzheng, an injection concocted from chinese medicinal herbs, combined with platinum-based chemotherapy for advanced non-small cell lung cancer: a systematic review. J. Exp. Clin. Cancer 2010, 29, 137.

[20] Chen, K. C.; Lu, R.; Iqbal, U.; Hsu, K. C.; Chen, B. L.; Nguyen, P.
A.; Yang, H. C.; Huang, C. W.; Li, Y. C.; Jian, W. S.; Tsai, S. H. Interactions between traditional Chinese medicine and western drugs in Taiwan: A population-based study. Comput. Meth. Prog. Biol. 2015, 122, 462-470.

[21] Baypinar, F.; Kingma, H. J.; van der Hoeven R. T. M.Becker M. L. Physicians' Compliance with a Clinical Decision Support System Alerting during the Prescribing Process. J. Med. Syst. 2017, 41, 96.

[22] van der Sijs H.; Aarts J.; Vulto A.Berg M. Overriding of Drug Safety Alerts in Computerized Physician Order Entry. J. Am. Med. Inform. Assoc. 2006, 13, 138-147.

[23] Desmedt S.; Spinewine A.; Jadoul M.; Henrard S.; Wouters D.Dalleur O. Impact of a clinical decision support system for drug dosage in patients with renal failure. Int. J. Clin. Pharm. 2018, 40, 1225-1233.

[24] Holstiege J.; Mathes T.Pieper D. Effects of computer-aided clinical decision support systems in improving antibiotic prescribing by primary care providers: a systematic review. J. Am. Med. Inform. Assoc. 2014, 22, 236-242.

[25] Verdoorn S.; Kwint H. F.; Hoogland P.; Gussekloo J.Bouvy M. L. Drug-related problems identified during medication review before and after the introduction of a clinical decision support system. J. Clin. Pharm. Ther. 2018, 43, 224-231.

[26] Liu Y. L.; Chu L. L.; Su H. C.; Tsai K. T.; Kao P. H.; Chen J. F.; Hsieh H. C.; Lin H. J.; Hsu C. C.Huang C. C. Impact of Computer-Based and Pharmacist-Assisted Medication Review Initiated in the Emergency Department. J. Am. Geriatr. Soc. 2019, 67, 2298-2304.

Received July 21, 2021 Accepted September 11, 2021 